\title{
ESTUDO RADIOGRÁFICO DA QUALIDADE DA OBTURAÇÃO ENDODÔNTICA POR ALUNOS DE GRADUAÇÃO EM ODONTOLOGIA
}

Radiographic study of endodontic root canal filling quality by undergraduate students in Dentistry

\author{
Stéphanie Quadros Tonelli ${ }^{1}$ \\ Thatyana Maldonado Nicácio Lafetá ${ }^{2}$ \\ Barbara Quadros Tonelli ${ }^{3}$ \\ João Vitor Quadros Tonelli ${ }^{4}$ \\ Manoel Brito-Júnior ${ }^{5}$
}

Resumo: Objetivo: Avaliar a qualidade de obturações endodônticas executadas por alunos em atividades laboratoriais da Disciplina de Endodontia de curso de graduação em Odontologia. Métodos: Foram avaliadas 80 obturações de canais radiculares em 35 dentes, por meio de radiografias padronizadas de dentes molares e pré-molares birradiculares. A qualidade da obturação foi criteriosamente avaliada em três parâmetros: i) limite apical, ii) homogeneidade e iii) conicidade, que foram estratificados em escores (E) 0,1 e 2. E0 e E1 corresponderam a acentuado e suave desvio da normalidade, respectivamente; enquanto E2 correspondeu ao padrão-ouro. Em função da combinação dos escores atribuídos, a obturação foi classificada em perfeita (PF), satisfatória (ST) ou deficiente (DF). Assim, com três escores E2: obturação PF; dois escores E2: obturação ST; e um ou nenhum escore E2: obturação DF. As associações entre as variáveis foram analisadas através do teste quiquadrado de Pearson $(\mathrm{p}<0,05)$. Resultados: As frequências de escores E2 (condição ideal) para

\footnotetext{
${ }^{1}$ Doutora em Odontologia Pontifícia Universidade Católica de Minas Gerais (PUCMinas), Belo Horizonte, MG - Brasil. $\bowtie$ stephanie tonelli@hotmail.com. 1 https://orcid.org/0000-0002-4671-9868.

${ }^{2}$ Graduanda do curso de Odontologia pelo Centro Universitário FIPMoc (UNIFIPMoc), Montes Claros, MG Brasil. $\bowtie$ thatyanalafeta@gmail.com. 1 https://orcid.org/0000-0002-7348-4929.

${ }^{3}$ Mestranda em Cuidados Primários em Saúde Universidade Estadual de Montes Claros (UNIMONTES), Montes Claros, MG - Brasil. $₫$ babi-tonelli@ hotmail.com. 1 https://orcid.org/0000-0003-3961-6374.

${ }^{4}$ Graduado em Odontologia pela Universidade Estadual de Montes Claros (UNIMONTES), Montes Claros, MG - Brasil. $\bowtie$ joaoqtonelli@gmail.com. 1 https://orcid.org/0000-0003-4723-996X.

${ }^{5}$ Doutor em Odontologia Restauradora e Professor do Departamento de Odontologia da Universidade Estadual de Montes Claros (UNIMONTES), Montes Claros, MG - Brasil. $\square$ manoelbritojr@gmail.com. https://orcid.org/0000-0002-3652-4047.
}

$\begin{array}{ccc}\text { Recebido em } & \text { Aceito em } & \text { Publicado em } \\ 23 / 01 / 2021 & 21 / 07 / 2021 & 30 / 09 / 2021\end{array}$


homogeneidade, limite apical e conicidade foram $97,5 \%, 87,5 \%$ e $73,8 \%$, respectivamente ( $>0,05$ ). Obturações PF, ST e DF ocorreram em 63,8\%, 33,8\% e 2,5\% dos casos, respectivamente. Não houve, portanto, associação estatisticamente significativa entre a distribuição dos escores dos parâmetros bem como da qualidade de obturação entre os grupos de dentes ( $>>0,05)$. Conclusão: As obturações realizadas apresentaram adequado padrão de qualidade, sendo a conicidade o parâmetro mais crítico, principalmente nos molares superiores.

Palavras-chave: Estudantes de Odontologia; Endodontia; Obturação do Canal Radicular; Radiografia.

Abstract: Objective: To evaluate the quality of endodontic obturations performed by students in laboratory activities of the Endodontic Discipline of undergraduate course in Dentistry. Methods: 80 root canal fillings were evaluated in 35 teeth by means of standardized radiographs of birradicular molar teeth and premolars. The quality of the obturation was carefully evaluated in three parameters: (i) apical limit, (ii) homogeneity and (iii) conicity, which were stratified into (E) 0,1 and 2. E0 and E1 corresponded to a marked and smooth deviation from normality, respectively; while E2 corresponded to the gold standard. Due to the combination of the assigned scores, the obturation was classified as either perfect (FP), satisfactory (ST) or deficient (DP). Thus, with three E2 scores: PF obturation; two E2 scores: ST obturation; and one or no score E2: DF obturation. The associations between variables were analyzed using the Pearson chi-square test ( $\mathrm{p}<0.05)$. Results: The frequencies of E2 (ideal condition) scores for homogeneity, apical limit and conicity were 97.5\%, 87.5\% and 73.8\%, respectively ( $p>0.05$ ). PF, ST and DF fillings occurred in $63.8 \%, 33.8 \%$ and $2.5 \%$ of the cases, respectively. There was therefore no statistically significant association between the distribution of the parameter scores as well as the quality of obturation between the groups of teeth ( $p>0.05$ ). Conclusion: The obturations performed presented an adequate quality standard, with taper being the most critical parameter, especially in the maxillary molars.

Keywords: Dental Student; Endodontics; Root Canal Obturation; Radiography.

\section{INTRODUÇÃO}

O ensino da prática endodôntica consiste, inicialmente, de instruções didáticas e pré-clínicas sobre princípios e técnicas fundamentais. Nas atividades laboratoriais os hábitos de trabalho são sedimentados permitindo a consolidação dos conteúdos teóricos ministrados, além de simular situações que serão vivenciadas no atendimento clínico futuro. Há uma 
estreita relação entre o aprendizado do laboratório e sua aplicação nas atividades clínicas diminuindo as dificuldades técnico-operatórias impostas pela complexidade anatômica do sistema de canais radiculares proporcionando capacitação do aluno e aprimorando a sua capacidade de resolução de problemas com vistas a alcançar o sucesso dos procedimentos realizados $^{1-4}$.

Considerando que a endodontia contemporânea abrange a completa limpeza, modelagem e a obturação hermética e tridimensional do sistema de canais radiculares ${ }^{3-6}$ a análise da qualidade de cada uma dessas etapas operatórias torna-se essencial para aprimoramento da visão critica acerca da técnica endodôntica ${ }^{5}$. Neste contexto, a obturação dos canais radiculares, reconhecida radiograficamente, tem sido a base de avaliação do tratamento endodôntico permitindo classificá-lo como sucesso ou insucesso ${ }^{2,7,8}$, bem como prestando-se para a análise e julgamento dos procedimentos técnicos adotados anteriormente $\mathrm{e}^{6,9-11}$.

De modo geral, o selamento efetivo do sistema de canais radiculares representa o coroamento do tratamento bem conduzido ${ }^{3}$, enquanto obturações deficientes, quase sempre, estão associadas com o fracasso da terapia empregada ao refletir a sanificação e formatação inadequadas, permitindo a permanência na estrutura dentária, de bactérias e seus subprodutos metabólicos ${ }^{12,13}$. Assim, a qualidade da obturação pode representar o desempenho das etapas operatórias que a antecedem, permitindo a avaliação de todo o tratamento realizado.

O presente estudo teve por objetivo avaliar a qualidade das obturações endodônticas executadas nas atividades laboratoriais na disciplina de Endodontia de um curso de graduação em Odontologia.

\section{MATERIAL E MÉTODOS}

Este estudo foi aprovado pelo Comitê de Ética em Pesquisa local sob protocolo número 632/2006.

A amostra foi constituída de dentes molares e pré-molares birradiculares tratados endodonticamente por alunos de graduação. Todos os dentes foram tratados usando técnicas padronizadas de preparo e obturação de canais radiculares. Empregou-se a técnica de instrumentação no sentido coroa-ápice, Biescalonada, coadjuvada pela irrigação com 
hipoclorito de sódio a 2,5\%. As obturações dos canais radiculares foram realizadas pela técnica da condensação lateral, utilizando cones de guta-percha acentuadamente cônicos, não padronizados, do tipo médium e fine-medium (Odous, Belo Horizonte, MG, Brasil) associados ao cimento à base de óxido de zinco e eugenol (Endofill, Dentsply Ind. e Com. Ltda., Petrópolis, RJ Brasil).

Os dentes foram incluídos em recipientes plásticos contendo mistura de gesso e serragem com o objeto de simular o alvéolo dentário. Foram obtidas radiografias padronizadas por meio da utilização de um dispositivo de acrílico que permitiu a realização das tomadas radiográficas com a mesma angulação vertical e horizontal e distância foco-filme de $40 \mathrm{~cm}$. Além disso, uma placa de acrílico capaz de simular a barreira de tecidos moles foi colocada em frente ao cilindro localizador de um aparelho de Rx (Dabi Atlante, Ribeirão Preto, SP, Brasil) operando a 70KVp e 8mA (Figura 1). O filme utilizado foi o do grupo D (KodakCo., Rochester, NY, EUA). As radiografias foram processadas pelo método tempotemperatura.

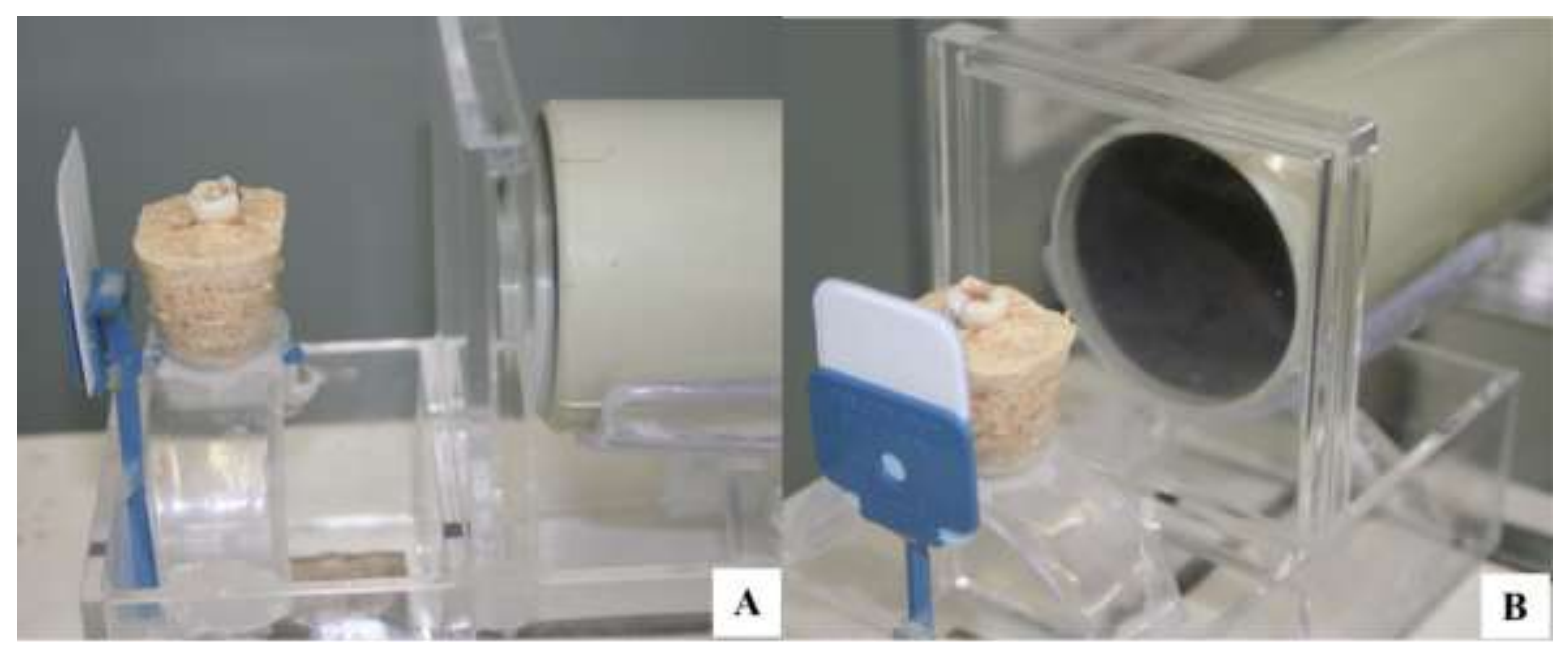

Figura 1: Dispositivo de acrílico para padronização das tomadas radiográficas dos espécimes incluídos em gesso e serragem.

Posteriormente, as radiografias foram projetadas em sala escura, com aumento de 10 vezes, sobre tela milimetrada e avaliadas por quatro examinadores. Para calibração dos examinadores foram utilizadas 36 radiografias de canais radiculares tratados endodonticamente de todos os grupos dentários. As avaliações foram realizadas com intervalo de pelo menos 1 semana e as incomuns discordâncias de interpretação foram decididas 
consensualmente. O teste Kappa foi aplicado para verificar as concordâncias intra e inter examinadores, obtendo-se elevados valores (K.> 0.8).

Conforme estudo prévio $^{14}$, a qualidade da obturação foi criteriosamente avaliada em três parâmetros: i) limite apical, ii) homogeneidade e iii) conicidade, os quais foram estratificadas em escores (E) 0,1 e 2. E0 e E1 corresponderam a acentuado e suave desvio da normalidade, respectivamente, enquanto E2 correspondeu ao padrão-ouro. Assim, com três escores E2: obturação perfeita; dois escores E2: obturação satisfatória; e um ou nenhum escore E2: obturação deficiente (Figura 2).

Os dados foram tabulados no programa SPSS (Statistical Package for the Social Sciences) 15.0 (IBM Corporation, Armonk, New York, Estados Unidos) for Windows. Para todas as variáveis foi determinada distribuição de frequência, sendo avaliada posteriormente a relação da qualidade das obturações e parâmetros radiográficos com os grupos dentários. Foi utilizado o teste estatístico quiquadrado de Pearson $(\mathrm{p}<0,05)$.

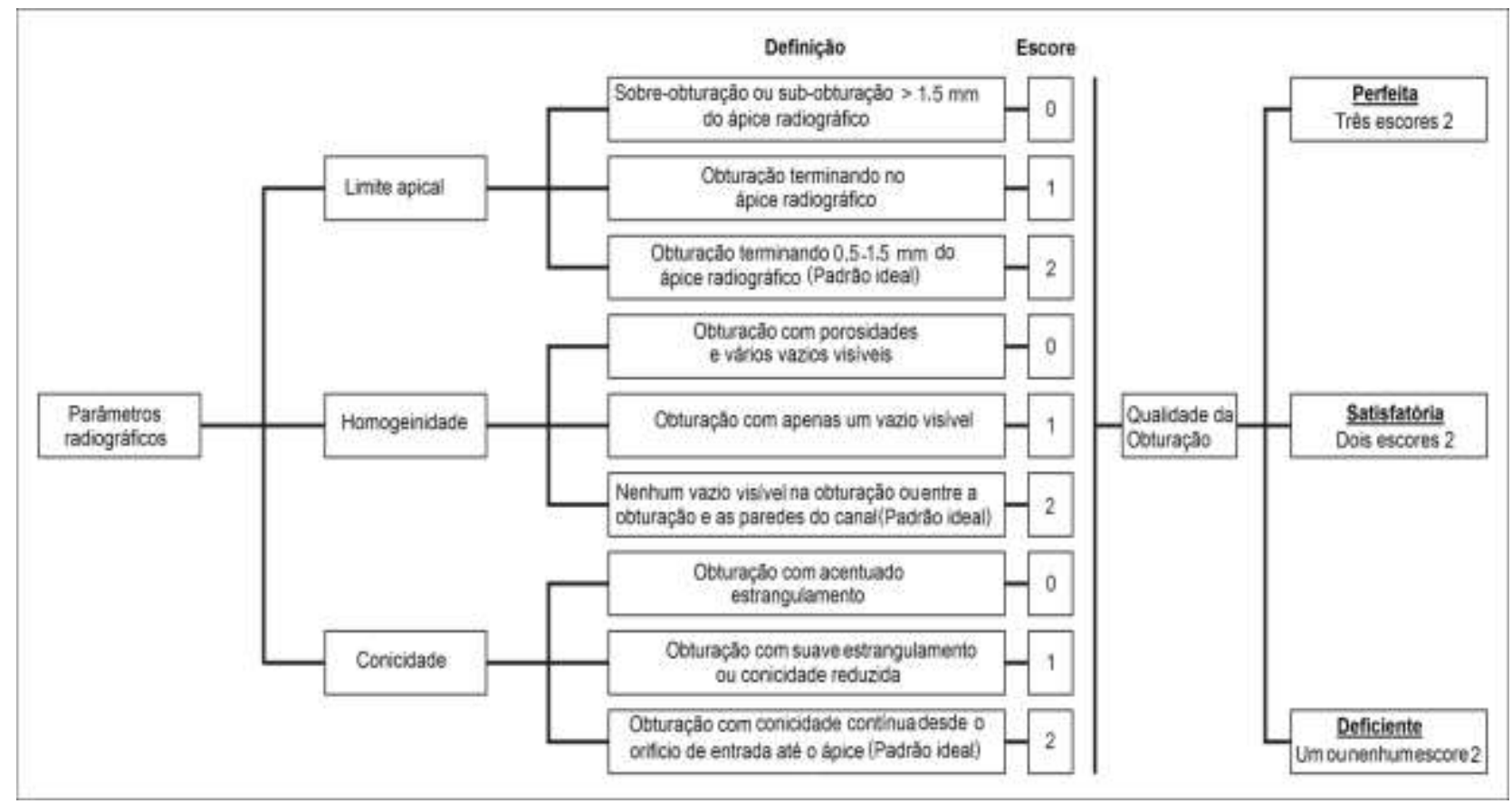

Figura 2: Representação esquemática da qualidade da obturação dos canais radiculares de acordo com os parâmetros radiográficos avaliados. Extraído de Santos et al. $(2010)^{14}$. 


\section{RESULTADOS}

Foram utilizados 35 dentes extraídos, totalizando 80 canais radiculares. A Tabela 1 mostra a distribuição dos canais radiculares obturados avaliados conforme o grupo de dentes.

Tabela 1. Distribuição absoluta e relativa dos canais radiculares obturados avaliados conforme grupo de dentes $(\mathrm{n}=80)$.

\begin{tabular}{lll}
\hline Canais radiculares & $\mathrm{N}$ & $\%$ \\
\hline Pré-molares & 10 & 12,5 \\
Molares Inferiores & 34 & 42,5 \\
Molares Superiores & 36 & 45,0 \\
Total & 80 & $100 \%$ \\
\hline
\end{tabular}

No que diz respeito aos parâmetros radiográficos, o limite apical e a homogeneidade receberam escores padrão-ouro em $87,5 \%$ e 97,5\% dos casos, respectivamente, demonstrando um padrão semelhante; já a conicidade apresentou a maior quantidade de desvios da normalidade, contudo, sem diferenças significativas $(p>0,05)$ quando comparado aos outros parâmetros (Figura 3). A qualidade da obturação foi considerada perfeita, satisfatória e deficiente em 63,8\%, 33,8\% e 2,5\% dos casos, respectivamente.

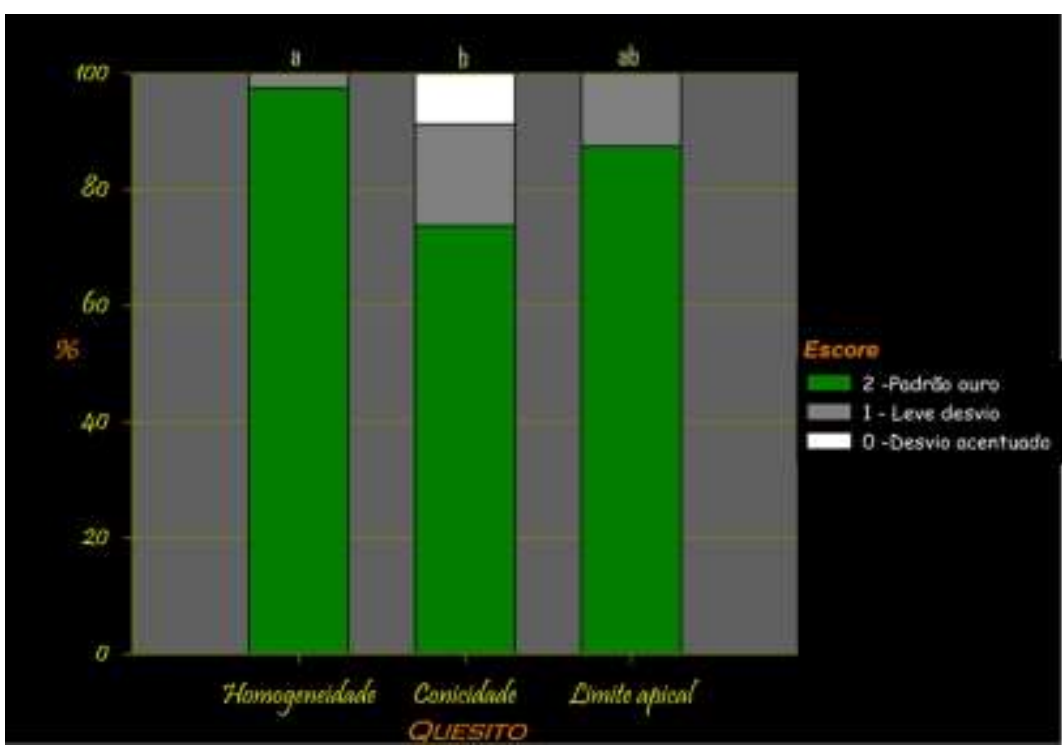

Figura 3: Distribuição dos escores de acordo com os parâmetros radiográficos avaliados em toda a amostra. Letras diferentes sobre as colunas significam diferenças significativas $(\mathrm{p}<0,05)$. 
Quando analisados os grupos dentários em função dos parâmetros radiográficos, foi verificada uma tendência, sem relação significativa $(p>0,05)$, dos molares superiores apresentarem a maior quantidade de desvios acentuados no parâmetro conicidade (Figura 4). Similarmente, considerando a relação grupo dentários e qualidade das obturações observou-se que os molares superiores apresentaram a menor quantidade de obturações perfeitas, com um percentual de 55,6\% quando comparados com os grupos de pré-molares e molares inferiores, que tiveram obturações perfeitas em 70,0\% e 70,6\%, respectivamente, sem diferenças significativas ( $>>0,05)$ (Figura 5).

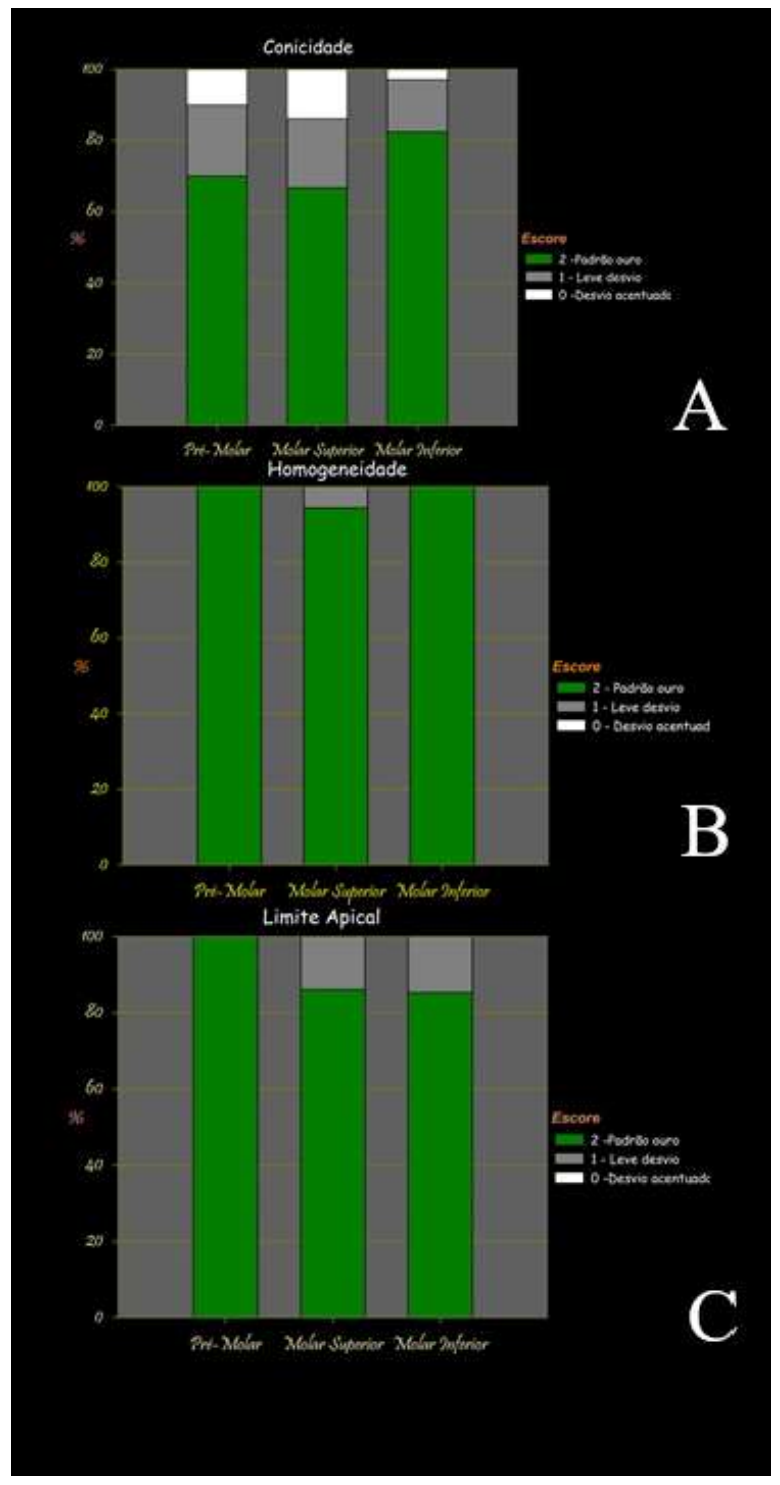

Figura 4. Frequência dos escores atribuídos aos parâmetros de qualidade das obturações em função dos dentes tratados. A. Conicidade; B. Homogeneidade e C. Limite apical. 


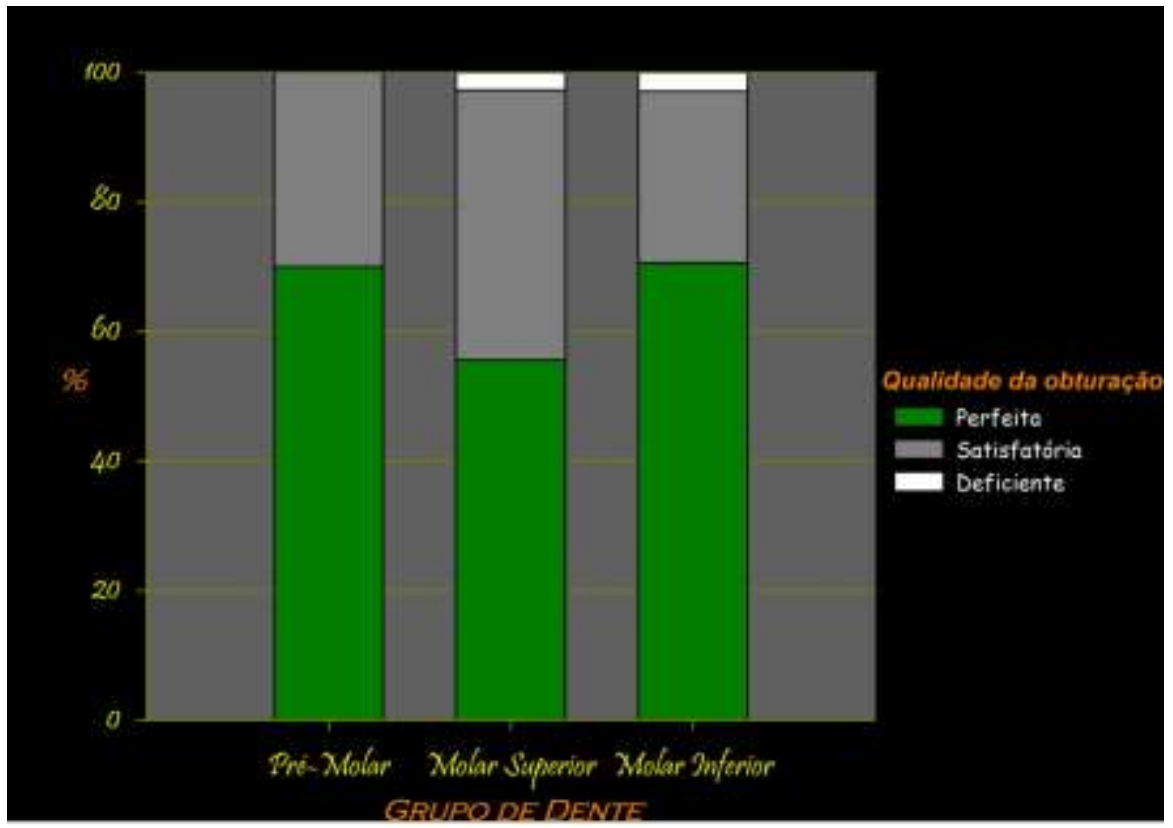

Figura 5. Distribuição da qualidade das obturações de acordo com os grupos dentários.

\section{DISCUSSÃO}

A avaliação da qualidade das obturações endodônticas neste estudo foi realizada por meio de radiografias periapicais, cuja principal limitação é apresentar imagem bidimensional de uma estrutura tridimensional ${ }^{14-17}$. A fim de minimizar erros de interpretação, as radiografias foram rigorosamente padronizadas e sua análise foi realizada por examinadores calibrados. Além disso, a metodologia adotada para avaliação radiográfica foi criteriosa e abrangente conforme demonstrado em estudo prévio ${ }^{14}$.

Os resultados deste estudo mostraram elevado percentual $(97,5 \%)$ de obturações endodônticas adequadas (perfeitas e satisfatórias) realizadas por alunos de graduação nas atividades laboratoriais em dentes morfologicamente mais complexos. Geralmente, na avaliação da qualidade da obturação endodôntica a maioria dos estudos considera apenas o limite apical (em geral, com valores da ordem de 0,5 a $2 \mathrm{~mm}$ aquém do ápice), e a homogeneidade da obturação (ausência de vazios ou de porosidades) como sendo referenciais do padrão-ouro, analisando-os, isoladamente ou em conjunto ${ }^{3,6,7,9,11}$. No atual estudo, os três parâmetros fundamentais da obturação do canal radicular foram analisados conjuntamente ${ }^{17-}$ ${ }^{20}$. Com metodologia semelhante, estudo radiográfico conduzido em laboratório de disciplina de graduação em Endodontia mostrou que $92 \%$ dos canais radicualres apresentaram adequada obturação, no entanto, nos molares este indice foi de $70 \%{ }^{3}$. No atual estudo, o favorável 
desempenho técnico dos estudantes de odontologia pode ser explicado pelo acompanhamento adequado por especialistas em endodontia, pertencentes ao corpo docente da universidade, na proporção de um professor para cada seis estudantes, durante todas as atividades laboratoriais.

Entre os parâmetros radiográficos da obturação avaliados no presente estudo, a homogeneidade atingiu maior índice de padrão-ouro (97,5\%). Esse achado é relevante, pois a presença de porosidades na obturação pode levar à infiltração marginal, favorecendo a reinfecção do canal radicular ${ }^{8,15}$.

O limite apical das obturações também apresentou elevado índice de adequação $(87,5 \%)$. Isso demonstra a assimilação correta, na graduação, deste requisito que, geralmente, é crítico para a obtenção de adequadas obturações ${ }^{14}$. Estudo anterior demonstrou um pior índice de adequação no limite apical, $72 \%{ }^{19}$. Deve-se ressaltar, entretanto, que durante as atividades laboratoriais do atual estudo os dentes extraídos foram manipulados sem uma estrutura de fixação, permitindo a visualização dos ápices dentários o que pode ter contribuído para o bom desempenho dos estudantes na determnação do limite apical.

Em relação à conicidade este foi o parâmetro que apresentou maiores problemas considerando toda a amostra, apresentando algumas situações de acentuados desvios. Em contraste, Fong et al. (2018) ${ }^{19}$ obtiveram resultado melhor com relação a este parâmetro, chegando a 97\% de obturações com conicidade adequada, porém, a partir da utilização de sistemas mecanizados de instrumentação. O uso da instrumentação mecanizada pode auxiliar numa instrumentação mais adequada e sua curva de aprendizado apresenta platôs mais elevados ${ }^{7}$. Ainda cabe observar que houve propensão dos canais de molares superiores, comparado aos molares inferiores, em apresentar maiores desvios na conicidade. No entanto, não constatou-se associação significativa pare este paramentro nos grupos dentários. Isso ocorreu, provavelmente, devido ao número limitado da amostra, de apenas 80 canais radiculares. Desta maneira, torna-se necessário um aumento do número de dentes em estudo futuros para a comprovação desse resultado.

Quando os grupos dentários foram comparados quanto à qualidade final das obturações, nos molares superiores foi verificado um menor índice de obturações perfeitas, com 55,6\% da amostra. Tal fato também foi comprovado em estudo prévio, em que a qualidade da instrumentação esteve relacionada à dificuldade do caso em si e não à técnica 
empregada ${ }^{16}$. Isso implica que os molares superiores devem demandar especial atenção nas atividades laboratoriais, bem como nos futuros atendimentos clínicos da graduação $^{21,22}$.

A clínica universitária constitui o primeiro contato dos estudantes de Odontologia com a prática da Endodontia, por isso se faz importante no treinamento clínico, visto que este treinamento está associado à melhoria da qualidade dos tratamentos endodônticos ${ }^{4,19,21,22}$. Portanto, é importante que novos estudos sejam conduzidos no sentido de se avaliar a qualidade da educação oferecida pelas instituições de ensino, observando e investigando os tratamentos realizados pelos alunos.

\section{CONCLUSÃO}

Concluiu-se que as obturações de canais radiculares apresentaram elevado padrão de qualidade. Dentre os parâmetros analisados, a conicidade apresentou o menor índice de adequação, principalmente nos molares superiores.

\section{REFERÊNCIAS}

1. Barrieshi-Nusair KM, Al-Omari MA, Al-Hiyasat AS. Radiographic technical quality of root canal treatment performed by dental students at the Dental Teaching Center in Jordan. J Dent 2004; 32(4):301-7.

2. Benenati FW, Khajotia SS. A radiographic recall evaluation of 894 endodontic cases treated in a dental school setting. J Endod 2002; 28(5):391-5.

3. Kamaura D, Carvalho GL, Lage-Marques JL, Antoniazzi JH. Avaliação do desempenho dos alunos de graduação durante a prática da técnica endodôntica. Rev Abeno 2003; 3(1):33-40.

4. Hendi SS, Karkehabadi H, Eskandarloo A. Iatrogenic Errors during Root Canal Instrumentation Performed by Dental Students. Iran Endod J 2018; 13(1):126-31. doi: 10.22037 / iej.v13i1.18507.

5. Jungnickel L, Kruse C, Vaeth M, Kirkevang LL. Quality aspects of ex vivo root canal treatments done by undergraduate dental students using four different endodontic treatment systems. Acta Odontol Scand 2018; 76(3):169-74. doi: 10.1080/00016357.2017.1396494.

6. Burke FM, Lynch CD, Ní Ríordáin R, Hannigan A. Technical quality of root canal fillings performed in a dental school and the associated retention of root-filled teeth: a clinical follow-up study over a 5-year period. J Oral Rehabil 2009 ; 36(7):508-15. 
7. Lynch CD, Burke FM. Quality of root canal fillings performed by undergraduate dental students on single-rooted teeth. Eur J Dent Educ 2006; 10(2):67-72.

8. Sonntag D, Delschen S, Stachniss V. Root-canal shaping with manual and rotary Ni-Ti files performed by students. Int Endod J 2003; 36:715-23.

9. Eleftheriadis GI, Lambrianidis TP. Technical quality of root canal treatment and detection of iatrogenic errors in an undergraduate dental clinic. Int Endod J 2005; 38:725-34.

10. Er O, Sagsen B, Maden M, Cinar S, Kahraman Y. Radiographic technical quality of root fillings performed by dental students in Turkey. Int Endod J 2006; 39(11):867-72.

11. European Society of Endodontology. Quality guidelines for endodontic treatment: consensus report of the European Society of Endodontology. Int Endod J 2006; 39(12):921-30.

12. Siqueira JF Jr, Roças IN, Alves FR, Campos LC. Periradicular status related to the quality of coronal restorations and root canal fillings in a Brazilian population. Oral Surg Oral Med Oral Pathol Oral Radiol Endod 2005; 100(3):369-74.

13. Sundqvist G, Figdor D, Persson S, Sjogren U. Microbiologic analysis of teeth with failed endodontic treatment and the outcome of conservative re-treatment. Oral Surg Oral Med Oral Pathol Oral Radiol Endod 1998; 85(1):86-93.

14. Santos SM, Soares JA, César CA, Brito-Júnior M, Moreira AN, Magalhães CS. Radiographic quality of root canal fillings performed in a postgraduate program in endodontics. Braz Dent J 2010; 21(4):315-21.

15. Al-Manei KK. Radiographic Quality of Single vs. Multiple-Visit Root Canal Treatment Performed by Dental Students: A Case Control Study. Iran Endod J 2018; 13(2):149-54. doi: 10.22037/iej.v13i2.19427.

16. Haug SR, Solfjeld AF, Ranheim LE, Bårdsen A. Impact of Case Difficulty on Endodontic Mishaps in an Undergraduate Student Clinic. J Endod 2018; 23. pii: S00992399(18)30212-7. doi: 10.1016/j.joen.2018.03.012.

17. Zambon da Silva P, Carlos Ribeiro F, Machado Barroso Xavier J Pratte-Santos R, Demuner C. Radiographic Evaluation of Root Canal Treatment Performed by Undergraduate Students, Part I; Iatrogenic Errors. Iran Endod J 2018; 13(1):30-36. doi: 10.22037/iej.v13i1.16800.

18. Moussa-Badran S, Roy B, Bessart du Parc AS, Bruyant M, Lefevre B, Maurin JC. Technical quality of root fillings performed by dental students at the dental teaching centre in Reims, France. Int Endod J 2008; 41:679-684.

19. Fong W, Heidarifar O, Killough S, Lappin MJ El Karim IA. An audit on technical quality of root fillings performed by undergraduate students. Int Endod J 2018; 51 Suppl 3:e197e203. doi: 10.1111/iej.12803. 
20. Santos SM, Soares JA, Costa GM, Brito-Júnior M, Moreira AN, de Magalhães CS. Radiographic parameters of quality of root canal fillings and periapical status: a retrospective cohort study. J Endod 2010; 36(12):1932-7.

21. Costa BE, Lima GM, Tonelli SQ, Nunes E, Silveira FF. Incidence and quality of root canal fillings in undergraduate dental students. RGO, Rev Gaúch Odontol. 2019;67:e2019.

22. Souza PO, Tonelli SQ, Lima MSFF, Sá PPA, Almeida CKM, Nunes E, Silveira FF.Abordagem clínico-radiográfica de dentes tratados endodonticamente: status periapical e qualidade das obturações e restaurações coronárias. Rev Norte Mineira de enferm. 2019; 8(1):77-83. 